\section{Mirror reversal simply explained without recourse to psychological processes}

\author{
TATSUO TABATA \\ Osaka Prefecture University, Sakai, Osaka, Japan \\ and \\ SHUICHI OKUDA \\ Osaka University, Ibaraki, Osaka, Japan
}

\begin{abstract}
This paper proposes a simple and definitive solution to the mirror reversal problem, "Why does a mirror reverse left and right but not up and down?" The solution is given by combining the inversion caused by the optical process of mirroring and the definition of the left-right axis. Thus the left-right reversal of mirror images essentially does not involve psychological processes, in contrast to the multiprocess hypothesis recently proposed by Takano.
\end{abstract}

Takano (1998) has proposed a multiprocess hypothesis to answer the mirror reversal problem, "Why does a mirror reverse left and right but not up and down?" In this problem, the mirror assumed is a single, plane mirror. His hypothesis has a difficulty, as will be described in the next section. With respect to the different hypotheses that have appeared in the past literature and to the criticisms of them, the reader should consult Takano's paper and Gregory's (1996) book.

The present paper describes a new, simple, definitive solution to the problem. The solution derives from the inversion caused by the optical process of mirroring and the definition of the left-right axis. Thus the left-right reversal of mirror images essentially does not involve psychological processes, in agreement with Gregory's argument but in sharp contrast to the multiprocess hypothesis.

\section{A Difficulty in the Multiprocess Hypothesis}

Takano (1998) has considered only two of the infinitely many orientations of an object with respect to a mirror. Namely, his analysis has been limited to the object that faces or is located sideways to the mirror. Then he concludes that reversal emerges psychologically for the former orientation, whereas it occurs optically for the latter. At what intermediate orientation is the psychological cause replaced by the optical? The multiprocess hypothesis does not seem to give an answer to this question. We here assert that this is a major defect of the multiprocess hypothesis.

\footnotetext{
We are grateful to Y. Takano, the author of the multiprocess hypothesis, and J. Kokame, professor emeritus of the University of Tokyo, for their kind communication and discussion. Thanks are due J. H. Hubbell for his useful comments in improving the text. Correspondence should be addressed to T. Tabata, Institute for Data Evaluation and Analysis, 198-51 Kami, Sakai, Osaka 593-8311, Japan (e-mail: tttabata@pearl.ocn.ne.jp).
}

\section{Preparation for the Solution}

We will begin the derivation of our solution with the following statement, which has been acknowledged by most authors who have studied the present problem:

1. A mirror reverses the direction of an object along the axis perpendicular to the mirror in producing its image.

Here and later in this paper, the word object is used to mean anything that can exhibit left-right reversal. Such a directional change as that described in the statement above causes a structural difference between the object and its mirror image (see, e.g., Gardner, 1964, 1990):

2 . The directional reversal in Statement 1 changes an asymmetric object into its enantiomorph (this change is called inversion, and the structural relation between the object and its mirror image is called enantiomorphism).

Statements 1 and 2 describe a purely physical phenomenon of inversion caused by mirroring, and similar explanations can be found in the textbooks of optics (see, e.g., Hecht, 1998; Meyer-Arendt, 1994). To assist the reader's understanding, we will explain the meanings of these statements by resorting to some examples.

Statement 1 means the following: When you are facing a mirror, your front-back axis is reversed in your image, which is facing you. However, the other two axes orthogonal to the front-back axis and each other (i.e., the topbottom ${ }^{1}$ and left-right axes of your body) are not reversed in your image with respect to the external world. When you are standing on the mirror, your top-bottom axis is reversed in your image, which is standing on its head, but the remaining two of your mutually orthogonal axes are not reversed in your image with respect to the external world.

Statement 2 means that the examples above can be paraphrased as follows: In the first example, your mirror image has the structure of your body, which has suffered such a physical transformation that the front of your body has pushed back through to the other side. ${ }^{2}$ In the second example, your image suggests that your body has undergone a similar transformation along the top-bottom axis.

Comparison of the two images in these examples shows that the structures of the images are the same. This is explained by the fact that the enantiomorph is obtained from an object by the reversal of any arbitrary axis (Bennett, 1970; Block, 1974). ${ }^{3}$ This characteristic of enantiomorphism can be understood by noting that the mirror image of the left hand, for example, has the structure of the right hand irrespective of its orientation relative to the mirror. ${ }^{4}$ Because of the generality of this fact, we can say:

3. The reversed structure of the mirror image (i.e., the enantiomorph) can be interpreted as having been obtained by the reversal of the object along any arbitrary axis.

In spite of this arbitrariness, however, we find this: If you have a watch around your left wrist, both of the mirror images in the aforementioned examples have one around the right wrist. Namely, both of the images are observed to be left-right reversed, irrespectively of the optical trans- 
formation axis. This reversal of the inherent left-right axis occurs not only for the images in the two different orientations of these examples, but also for the image of any object in any orientation with respect to the mirror. ${ }^{5}$ The fundamental reason for this universal occurrence of left-right reversal is what we describe below.

Statement 3 and the observation in the paragraph above reduce the mirror reversal problem to finding the solution to this question: Why, in the recognition of an enantiomorph, is the left-right axis the axis that is reversed by inversion among other possible axes? Gardner attributed the reason to verbal convention stemming from our bilateral symmetry. Though some authors have supported this explanation (Bennett, 1970; Corballis \& Beale, 1976; Hofstadter \& Dennett, 1981), we think that there is a stronger reason.

\section{Solution}

We note the following fact related to the definition of left and right:

4. The left-right axis of an object (or its mirror image) is determined by a specific 3-D relation with respect to the top-bottom and front-back axes inherent in the object (or its mirror image), after the latter two axes have been determined by the use of externally observable asymmetry as a criterion for the sense of axes. ${ }^{6}$

The essence of this statement is that left and right cannot be defined until the top-bottom and front-back axes have been defined. Consider how we define left and right in a car, for example, even without being conscious of the definition above. A car does not have a heart by which most of us can remember its left side. A steering wheel may be on the left or right, depending on the traffic laws of a country. However, the top-bottom and front-back axes of the car are evident from its shape. We first align these directions (at least in our imagination) with those of our bodies, and then find the left and right of the car. The left and right of a mirror image are determined similarly by treating the image like a real object. Further evidence for the fact of definition given above will be presented in the next section. With regard to the "specific 3-D relation" in Statement 4, different prescriptions equivalent to each other can be made, and an example will also be given in the next section.

The topside of an object is commonly defined as the side located upward ${ }^{7}$ in its natural posture. Concerning the front-back direction, Mayo (1958) gave three criteria for its determination: The front direction is (1) the direction of "facing" or "looking," (2) the direction of normal movement, or (3) the direction of the object normally facing the viewer. "Externally observable asymmetry" in Statement 4 is meant to include all reasonable clues and criteria to determine the sense of the top-bottom and front-back axes. Without any asymmetry, we can hardly define which is the front end, for example, of a streetcar that is standing still. Only when the street car is running can movement break symmetry, so that we can define its front-back direction. ${ }^{8}$

When Statements 1-3 are considered together with Statement 4, we reach the following conclusion:

5. Among the top-bottom, front-back, and left-right axes, the first two cannot be reversed because of their preceding determination from external asymmetry. Consequently, the left-right axis has to be identified as the axis reversed in inversion by mirroring.

For example, the topside of a person's image is defined by his or her head, and the front side, by his or her face. We cannot really imagine one's head as defining the bottom of the top-bottom axis or one's face as defining the back of the front-back axis. No matter how the body is optically transformed, the head is taken to define the top and the face is taken to define the front. To determine the left or the right of the inherent left-right axis, by contrast, we rely not on any asymmetry along this axis but on the predetermined top-bottom and front-back axes. Namely, the left-right axis is a derivative axis that is defined only after the other two axes have been defined. Thus the reversal that has produced the enantiomorph is necessarily attributed to the left-right axis independently of the orientation of the person with respect to the mirror. We can apply a similar explanation also for alphanumeric characters and other objects (see note 5). Thus Statements 1-5 give a solution to the mirror reversal problem in every conceivable case.

\section{Discussion}

One of the most general definitions of the word left (adjective) found in dictionaries is the following:

1. on or towards the side of the human body which is to the west when facing north. 2 . on or towards the part of an object which is analogous to a person's left side or (with opposite sense) which is nearer to a spectator's left hand.

(Thompson, 1995)

The simple phrase facing north in Definition 1 means that the human stands in the natural manner (this defines the top-bottom axis) with the front side directed to the north (this refers to the front-back axis). Thus this description implies that the top-bottom and front-back axes have to be predetermined, supporting Statement 4 . It also gives an example of the "specific 3-D relation" referred to in the same statement. This subordination of the leftright axis is thought to have originated from the approximate bilateral symmetry of humans, animals, and many man-made things, as Gardner $(1964,1990)$ has described.

The necessity of the predetermination of the topbottom and front-back axes is also evident from the following. Consider a spherical or a cylindrical object without any pattern or mark on its surface. Such an object has neither the top-bottom axis nor the front back axis inherent in it, so that we cannot speak of its left-right axis either.

In his review of the mirror reversal problem, Gregory (1996) asks how the asymmetric result of left-right re- 
versal arises from a mirror, which is optically symmetric for the exchange of the left-right and top-bottom axes. However, he does not give a satisfactory answer to this question. The present solution indicates that the asymmetry in the definition between left-right and top-bottom axes is the source of the asymmetric result.

Either the precedence of top-bottom and front-back in the determination of the three axes or other, similar concepts have already been mentioned by Bennett (1970), Takano (1998), Block (1974), and Takizawa (1964). However, the first two authors did not apply this principle to the full explanation of the mirror reversal problem, and the statements of the latter two authors are not explicit in this regard. Bennett's main theme was enantiomorphism, and he used the definition of left and right only to explain the relation of an object and its enantiomorph; with respect to the mirror reversal problem, he simply accepted Gardner's verbal convention hypothesis. Takano adopted the order of axis determination only to explain the rotation of the representational frame about a vertical axis in a class of reversal. Block wrote an example of specifying the right side of an arbitrary object that had a bottom and a front, and concluded that the left-right reversal by a mirror was due to a difference between the two types of direction, thus failing to mention explicitly the crucial point made here. In the solution section of his textbook on algebra and geometry, Takizawa gave a short explanation only for the latter half of the mirror reversal problem ("Why does a mirror not reverse up and down?") and mentioned that the left-right axis was determined after the front-back axis but that the top-bottom axis was determined independently of the front-back axis. Because of the brevity of his explanation, it is not clear whether he had the same solution as ours concerning the first half of the problem or whether his solution was similar to Gardner's verbal convention hypothesis.

The present solution has been derived only from the optical process of producing the enantiomorph of an object (i.e., inversion) and the definition of the left-right axis; it is applicable to any orientation of the object with respect to the mirror. This derivation does not indicate that the left-right reversal of the mirror image involves psychological processes in an essential manner. ${ }^{9}$ This is in agreement with Gregory's (1996) statement that "mirrorreversal is not a psychological turnabout, or a problem of description," but it is in sharp contrast to Takano's multiprocess hypothesis. Though his paper includes important criticisms of the past literature, Takano's hypothesis is too restrictive, and it is complicated by psychological processes that are unnecessary.

\section{REFERENCES}

Bennett, J. (1970). The difference between right and left. American Philosophical Quarterly, 7, 175-191.

BLOCK, N. J. (1974). Why do mirrors reverse right/left but not up/down? Journal of Philosophy, 71, 259-277.

Corballis, M. C., \& BEALE, I. L. (1976). The psychology of left and right. Hillsdale, NJ: Erlbaum.
Gardner, M. (1964). The ambidextrous universe. New York: Basic Books

GaRDNER, M. (1990). The new ambidextrous universe (3rd rev. ed.). New York: W. H. Freeman.

GLEICK, J. (1992). Genius. London: Little Brown.

Gregory, R. (1996). Mirrors in mind. New York: W. H. Freeman.

Hecht, E. (1998). Optics (3rd ed.). Reading, MA: Addison-Wesley.

Hofstadter, D. R., \& DennetT, D. C. (1981). The Mind's $I$. New York: Basic Books.

Mayo, B. (1958). The incongruity of counterparts. Philosophy of Science, 25, 109-115.

MEYER-ARENDT, J. R. (1994). Introduction to classical and modern optics (4th ed.). Englewood Cliffs, NJ: Prentice-Hall.

ShePARD, R. N., \& METZleR, J. (1971). Mental rotation of threedimensional objects. Science, 171, 701-703.

TAKANO, Y. (1998). Why does a mirror image look left-right reversed? A hypothesis of multiple processes. Psychonomic Bulletin \& Review, $5,37-55$.

TAKIZAWA, S. (1964). Saishin daisugaku to kikagaku [Newest algebra and geometry]. Tokyo: Hirokawa Publishing.

ThOMPSON, D. F., ED. (1995). The concise Oxford Dictionary of current English (9th ed.). Oxford: Oxford University Press, Clarendon Press. Tomonaga, S. (1965). Kagaminonaka no sekai [The world in the mirror]. Tokyo: Misuzu-Shobo.

\section{NOTES}

1. We use top and bottom instead of up and down. The reason is as follows: "Up and down" as well as "left and right" in the mirror reversal problem are considered to refer to the directions inherent in the object or its mirror image. Thus "top and bottom" is more appropriate than "up and down."

2. John Wixted (personal communication, 1999) suggested this explanation. Richard Feynman denied a similar explanation and arrived at a conclusion different from ours, as quoted by Gleick (1992), "We cannot image ourselves 'squashed' back to front, so we imagine ourselves turned left and right, as if we had walked around a pane of glass to face the other way. It is in this psychological turnabout that left and right are switched." Criticizing Feynman's words, Gregory (1996) wrote, "We generally appear squashed rather flat in a photograph," adding that psychological turnabout might not be entirely clear. Interestingly, Sin-Itiro Tomonaga, who shared the 1965 Nobel Physics Prize with Feynman and Julian Schwinger, also discussed the mirror reversal problem with his colleagues in his young days, and they thought that the top-bottom and front-back axes had absoluteness in a "psychological space" (Tomonaga, 1965).

3. The reversal of all three orthogonal axes at the same time (this procedure is called parity operation in physics) also produces an enantiomorph

4. If one does not like the words left and right in the explanation of enantiomorphs, one may consider mirroring one of Bennett's boxes, A and B (Bennett, 1970; Corballis \& Beale, 1976). These are essentially the same rectangular parallelepipeds, with a minor difference. Both $A$ and $B$ have a corner sliced off, but the location of the sliced-off corner of $B$ is next to the corner that corresponds to the sliced-off corner of $A$ to make $\mathrm{A}$ and $\mathrm{B}$ enantiomorphs (a drawing of the boxes may be found in the references given above).

5. We do not commonly speak of inherent left or right for alphanumeric characters, but "the right of a character" means the side of the character nearer to "our" right side when we face it. However, we can assign the inherent left-right axis also for alphanumeric characters and other objects for which we do not do so conventionally. This assignment makes the present explanation applicable to such objects too. The inherent left and right are simply the right and left conventionally spoken of for such objects.

6. For an alphanumeric character, the front-back ax is of the sheet or the surface layer on which the character is written is regarded as the front--back axis referred to in Statement 4.

7. The up-down axis is meant here as an axis of a framework attached to the thing external to the object in consideration. In most cases, 
the external thing is the earth and "up" is away from the center of the earth (Block, 1974). When an object is an alphanumeric character, however, we more often have to consider our field of vision instead of the earth, because we put a sheet of paper horizontally for reading characters on it.

8. "Externally observable asymmetry" is mostly along the axis that we are going to define, but there are exceptions. When the object is the vertically symmetric character $D$ or $E$, for example, we do not define its top and bottom by vertical asymmetry (because there is no such asymmetry), but we compare its two-dimensionally asymmetric pattern with its standard image, which has the predetermined top-bottom axis in our memory.

9. One of the reviewers of the present paper suggested that the psychological process of mental rotation studied by Shepard and Metzler (1971) plays a role in apprehending the left-right reversal of a mirrored object. However, the authors consider that the apprehension of leftright reversal and reversal itself are different things.

(Manuscript received September 15, 1998 revision accepted for publication April 28, 1999.) 